ARTICLE

\title{
Managing risk in the face of adversity: design and outcomes of rapid glaucoma assessment clinics during a pandemic recovery
}

\author{
Hari Jayaram (iD ${ }^{1,2,3^{凶}}$, Alex J. Baneke ${ }^{1}$, Joy Adesanya ${ }^{1}$ and Gus Gazzard (D) ${ }^{1,2,3}$ \\ (c) The Author(s), under exclusive licence to The Royal College of Ophthalmologists 2021
}

BACKGROUND: The provision of timely care to the high volume of glaucoma patients stratified as "low risk" following pandemicrelated appointment deferrals continues to prove challenging for glaucoma specialists. It is unknown whether stratification as "low risk" remains valid over time, raising the potential risk of harm during this period if left unmonitored. This study aimed to evaluate whether Rapid Glaucoma Assessment Clinics (RGACs) are an effective method of assessing "low-risk" patients in order to identify those who may need an escalation of care, therefore reducing the risk of the future incidents of preventable vision loss. METHODS: RGACs were developed which comprised a brief advance telephone history by a clinician and then ophthalmic technician-measured visual acuity and intraocular pressure in clinic. We report outcomes from the first month of operation describing attendance patterns, the proportion of patients from this "low risk" cohort requiring escalation and underlying reasons for treatment escalations.

RESULTS: 639 patients were invited to attend RGACs. 75\% attended their booked appointment. Pre-attendance telephone consultations were associated with lower non-attendance rates ( $13.9 \%$ vs $29.3 \%, p<0.00001)$. $15 \%$ of patients were no longer deemed to remain at "low risk" with further expedited clinical review scheduled. $10.4 \%$ of patients required an escalation in treatment following review.

CONCLUSIONS: RGACs are an effective approach to deliver high throughput clinical assessments for large numbers of "low-risk" glaucoma patients with deferred appointments. They enable the rapid identification and treatment of patients who would otherwise face significantly delayed review reducing the risk of future preventable vision loss.

Eye (2022) 36:1799-1803; https://doi.org/10.1038/s41433-021-01738-0

\section{INTRODUCTION}

Glaucoma is the leading cause of preventable sight loss in the United Kingdom and is responsible for almost a third of new certifications of visual impairment [1]. The delivery of high-quality glaucoma care across the United Kingdom was a severe challenge even prior to the COVID-19 pandemic with increasing demands on clinical services: The Royal College of Ophthalmologists (RCOphth) "The Way Forward" report projected a $44 \%$ increase in the number of people in the UK with glaucoma by 2035 [2]. Delayed follow up and a lack of clinic capacity continued to contribute to cases of permanent and severe vision loss $[3,4]$, which led to a Healthcare Safety Investigation Branch report released prior to the pandemic highlighting the lack of timely monitoring for glaucoma patients and the need for service redesign [5].

This pre-existing challenge has only been magnified by the recent COVID-19 pandemic which will undoubtedly influence how we deliver care to glaucoma patients in the years ahead [6]. The challenge facing all glaucoma units within the United Kingdom is how to safely manage the significant numbers of appointments that were deferred as a consequence of the pandemic, in the context of constrained resources, anticipated fiscal austerity and the need for ongoing social distancing measures.
Risk stratification is integral in identifying and prioritising which patients need to be seen soonest. However there continues to be no nationally agreed, evidence-based risk stratification model for glaucoma care and considerable variation in approaches exists between eye units within the United Kingdom. At Moorfields, we have been able to see patients stratified as "high risk" or "medium risk" over the past year. However, delivering timely care to those labelled as "low risk" continues to prove a significant challenge with appointments for this group of patients having the longest deferral period by definition. Risk stratification is necessarily based upon historical clinical data. It is not known what proportion of patients classified as "low risk" or "stable", based on data captured up to twelve months or more prior, would in fact remain in this stratum over time, leading to the risk of undetected vision loss during this period if left unmonitored.

Rapid Glaucoma Assessment Clinics (RGACs) were therefore developed to provide a high-throughput approach to manage this large cohort of patients previously classified as "low risk" and otherwise subject to significant further deferral, with capture of the current minimum clinical data (visual acuity and intraocular pressure (IOP)) required for rudimentary glaucoma risk stratification. This study aimed to evaluate whether RGACs are an effective

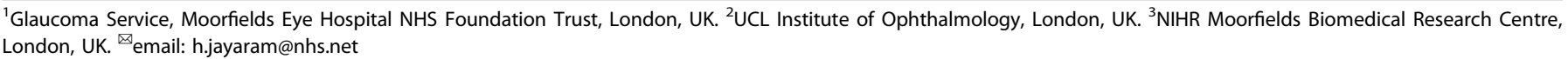

Received: 28 April 2021 Revised: 16 July 2021 Accepted: 29 July 2021

Published online: 10 August 2021 
Table 1. Moorfields blended risk stratification approach to identify suitable patients for RGACs.

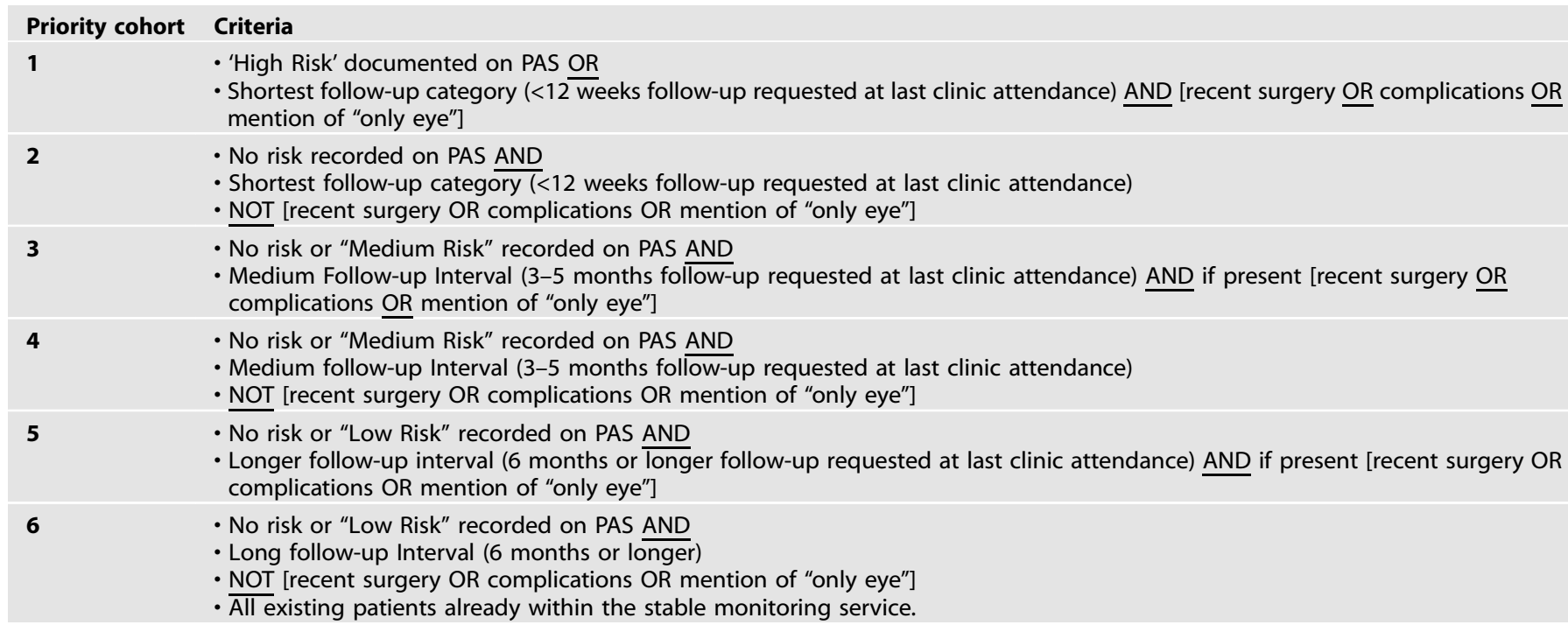

PAS Patient Administration System.

method of performing high throughput, data-driven risk restratification of patients thought to be at low-risk to identify those in need of escalation of care, to reduce the risk of preventable vision loss.

\section{MATERIALS AND METHODS}

This study was registered and approved by the Clinical Audit and Assessment Committee at Moorfields Eye Hospital (Ref: CA20/GL/750).

Risk stratification was performed by the Performance \& Information department at Moorfields, through a blended approach from the trust outpatient waiting list using (i) pre-existing documented risk stratification (high/medium/low) within the Patient Administration System (PAS, Silverlink Software, UK); (ii) previously planned follow-up intervals; and (iii) data from free text entries within the OpenEyes electronic medical record (EMR). Details of this process are summarised in Table 1. Patients stratified into priority cohort 6 , therefore those deemed to be at the lowest risk, were suitable to be booked into the RGACs. Within this cohort, patients were booked in order of the longest waiting category i.e. those waiting longest since their last clinic attendance were booked first. Patients known to have dementia, significant learning disability and those hard of hearing were seen through conventional clinical pathways.

Following screening for eligibility and a telephone call to confirm the appointment booking by administrative staff, patients were briefly telephoned by a member of the clinical care team (specialist optometrist or clinical fellow) prior to their attendance to record a brief interval history on the EMR. This included asking if any concerns had arisen since the last attendance and clarification of eye drop usage and any associated problems. It was also made clear that (i) attendance at the RGAC was for brief screening only to ensure patients are monitored following the delays caused by the pandemic and would not include visual field testing or optic nerve imaging; (ii) the tests will be performed by a technician and that they would not see a doctor on the day; and (iii) should any concerns arise from the data collected, a further telephone consultation would be performed by a member of the clinical team within 3 working days of the appointment.

RGACs were scheduled on Saturdays in order to generate additional clinic capacity, with appointments scheduled at 10-minute intervals, and staffed only by ophthalmic technicians. On the day of attendance, technicians measured patients' best-corrected visual acuity (unaided or spectacle-corrected, pinhole) using a 6 metre Snellen Chart and IOP using the Ocular Response Analyser (ORA, Reichert Technologies, Depew, NY, USA). Visual Acuity, ORA IOPg, ORA IOPcc and waveform score were then recorded on the EMR. Clinical review was performed by a glaucoma consultant within 3 working days of the attendance $(\mathrm{HJ})$. If no concerns arose regarding patient's visual acuity or intraocular pressure during review, a further formal review was scheduled for a nine-month interval in the stable monitoring service ("virtual clinic").

In this report we evaluate the performance of the of the first month of operation of RGACs at Moorfields Eye Hospital in October 2020. We aimed to identify the overall attendance pattern for the RGACs and to investigate whether this differed between those who received a pre-visit telephone consultation and those who did not (e.g. unavailable when called, incorrect telephone number on PAS). In addition, for those who chose not to attend we examined the proportion who telephoned the clinic to cancel and defer appointments due to concerns about attending during the pandemic.

We also aimed to establish the proportion of patients from this cohort stratified as "low risk" who in fact needed an escalation in care according to three criteria, (i) further treatment for raised/uncontrolled IOP (e.g. a need for change in medication or scheduling for selective laser trabeculoplasty); (ii) further investigation due to a reduction in visual acuity by $\geq 2$ lines of Snellen Acuity compared to their last attendance; and (iii) a clinical decision for expedited formal review sooner than 4 months or in a consultant led face-to-face clinic.

Outcomes from RGAC attendances were collated using Microsoft SQL Server Reports Software, combining data from our EMR and PAS systems. Individual patient encounters were reviewed manually within the EMR in order to confirm the attendance, outcome and clinical management plan. Booking outcomes were classified according to the following categories: (i) Attended; (ii) Chose not to attend; and (iii) Telephoned to Cancel. Clinic outcomes were categorised into the following groups: (i) Routine stable monitoring service follow up in 9 months; (ii) Specified follow-up interval $\leq 4$ months and (iii) Specified follow-up interval $\leq 2$ months. The underlying reasons for treatment escalation and details of the proposed escalation plan discussed by telephone with patients were also noted and extracted into Microsoft Excel. The Chi-Squared test was used to determine the significance of any differences between categorical observations. Other statistical comparisons were made with an unpaired $t$-test and one wayANOVA with correction for multiple comparisons where appropriate.

\section{RESULTS}

Six hundred and thirty-nine eligible patients were invited to attend RGACs on four Saturdays during the first month of operation in October 2020 . This equated to 160 patients per clinic day. The mean interval from last follow-up was 14.8 months (95\% Cl: 14.5-15.0).

\section{Attendance patterns for RGACs}

Seventy-five percent of patients attended their booked appointment (Table 2). Of those who did not attend, $21.4 \%$ chose not to attend on the day and $3.6 \%$ telephoned in advance to cancel and 
Table 2. Summary of booking outcomes.

\begin{tabular}{lc}
\hline Total patients booked & Number (\%) \\
\hline Attended & $639(100)$ \\
\hline Telephoned to cancel & $479(75.0)$ \\
\hline Chose not to attend & $23(3.6)$ \\
\hline
\end{tabular}

rebook their appointment at a later date due to concerns about attending during the pandemic. There was no significant difference in the mean interval from the last follow-up between patients who attended (14.5 months, $95 \%$ Cl: 14.2-14.8), chose not to attend on the day (15.6 months, $95 \% \mathrm{Cl}: 14.9-16.2)$ and those who telephoned to rebook (15.3 months, 95\% Cl: 13.5-17.1). Despite attempts to conduct a brief pre-attendance telephone consultation with all booked patients, only $54 \%$ were contactable by telephone in advance of their booked appointment. The proportion of patients who chose not to attend on the day was significantly lower in the group that received a pre-attendance telephone consultation compared to those who could not be contacted $(13.9 \%$ vs $29.3 \%, p<0.00001)$. Receiving a preattendance telephone consultation was also associated with patients being more likely to telephone the clinic to inform them of their choice not to attend in advance of their appointment $(6.1 \%$ vs $0.7 \%, p<0.0003)$ which allowed other patients to be scheduled therefore improving clinic efficiency (Table 3 ).

\section{Clinic outcomes - next follow-up interval}

Amongst patients who attended their appointment, $82.3 \%$ were deemed to remain at "low risk" on the basis of the updated clinical information gathered, in the context of their previous clinical records. These patients were therefore booked for further review in the stable monitoring service with perimetry and optic nerve imaging, within a nine-month follow-up interval (Table 4). However, $15 \%$ of patients were no longer deemed to remain at "low risk" following review with further formal assessment scheduled within a 4-month interval, and with $2.7 \%$ judged to require further review in a face-to-face clinic within 2 months. The latter two groups of patients had a greater mean interval from prior follow up than those deemed to remain at "low risk" (14.8 months vs 14.5 months) although this was not statistically significant.

\section{Observations indicative of increased risk and treatment escalations}

Amongst the 479 patients that attended, 50 patients (10.4\%) demonstrated evidence of increased clinical risk requiring an escalation in treatment. 23 patients $(4.8 \%)$ were found to have uncontrolled IOP (IOP $>30 \mathrm{mmHg}$ ) and 12 patients $(2.5 \%)$ were noted to have lost 2 lines or more of best-corrected Snellen Visual Acuity in either eye since their last clinic attendance (Table 5). Analysis of treatment escalations showed that 29 patients $(6.1 \%)$ required a change or increase in their glaucoma medications, 13 patients $(2.7 \%)$ were scheduled for selective laser trabeculoplasty in order to lower IOP, 5 patients $(1.0 \%)$ were scheduled for cataract surgery and 3 patients $(0.6 \%)$ were scheduled for glaucoma surgery (Table 5).

\section{DISCUSSION}

Approximately 40,000 glaucoma outpatient appointments were deferred across the Moorfields Network at the start of the pandemic in March 2020. We were able to rapidly reconfigure our services to continue to see patients stratified as "high risk" or medium risk within face-to-face clinics with restricted capacity and through a rapid expansion of our existing outpatient-based diagnostic clinic pathways, commonly referred to as "virtual clinics" $[7,8]$. The current national guidance from the RCOphth however has very restricted patient eligibility for virtual clinics [9]. In order to see patients at medium-high risk of glaucoma-related visual impairment in a timely manner, we significantly relaxed these criteria in conjunction with clinical review and telephone consultations performed by fellowship trained glaucoma specialists. However, despite this rapid service expansion, a major challenge arose in how to manage the backlog of over 25,000 patients whose appointments had been deferred and were classified as "low risk" through our blended risk stratification approach (Table 1) and who therefore would therefore not be scheduled to be seen for a considerable period of time.

Increased demand for clinic capacity has a significant impact upon service delivery within NHS ophthalmic services and inevitably increases the risk of clinical incidents related to preventable vision loss associated with delayed care [5]. The objective of risk stratification in the context of glaucoma care is to ascertain which patients are at the highest risk of sight loss. The RCOphth and the United Kingdom \& Eire Glaucoma Society released details of a potential such tool ('Glauc-Strat-Fast') in July 2020 [10]. This approach and others such as the blended approach used at Moorfields can be utilised to identify which patients ought to be prioritised, and those who can be considered as "low risk". The patient types classified as the lowest risk (Green Stratum) by 'Glauc-Strat-Fast' (e.g. Moderate Primary Open Angle Glaucoma, Ocular Hypertension, Glaucoma Suspects) are identical to those within the sixth cohort of our blended approach eligible for RGACs, which comprised the overwhelming majority of deferred appointments.

The accuracy of any risk stratification strategy is dependent upon the extent and precision of the available clinical information used to inform decision making. Patients at medium-high risk will be prioritised and hopefully reviewed in a timely manner. However, the assumption that a patient categorised as "low risk" based upon clinical parameters obtained 12 months ago will remain as "low risk" for potentially a further 12 months, is hazardous. Our study found that fifteen percent of patients stratified as "low risk" were no longer judged to fall in this stratum following clinical review of historical records, current visual acuity and IOP measurements and in fact required expedited formal review. Over ten percent of "low risk" patients required an immediate escalation in treatment, and therefore if appointments had been deferred according to risk stratification, this may have led to a significant risk of preventable vision loss in this cohort. Considering that the "low risk" cohort comprised over 25,000 patients at Moorfields alone, it can be extrapolated that over 2500 of these may in fact be at higher risk of preventable glaucomarelated vision loss, emphasising the challenge being faced by glaucoma units across the United Kingdom.

An increased use of remote consultations is likely to be a lasting legacy from the COVID-19 pandemic. Over the past year, this

Table 3. The impact of receiving a pre-attendance telephone call on non-attendance.

\begin{tabular}{|llll|}
\hline & Total (\%) & Chose not to attend (\%) & Telephoned to cancel (\%) \\
\hline Received pre-attendance telephone call & $345(54.0)$ & $48(13.9 \%)$ & $21(6.1 \%)$ \\
\hline Did not receive pre-attendance telephone consultation & $287(44.9)$ & $84(29.3 \%)$ & $2(0.7 \%)$ \\
\hline No information available about pre-attendance telephone consultation & $7(1.1)$ & - & - \\
\hline$p$-value (Chi-squared) & & $<0.00001$ & $<0.0003$
\end{tabular}


Table 4. Summary of clinic outcomes.

\begin{tabular}{|lc|}
\hline Total number of patients attended & Number (\%) \\
\hline Routine stable monitoring service: 9 months & $479(100)$ \\
\hline Expedited formal follow up: $\leq 4$ months & $407(85.0)$ \\
\hline Expedited formal follow up: $\leq 2$ months & $72(15.0)$ \\
\hline
\end{tabular}

modality has been embraced across medical specialities with the development of national guidance by NHS England and the National Institute for Health \& Care Excellence [11]. High levels of patient satisfaction with remote consultations have been demonstrated within ophthalmology [12] and this should serve as a springboard to further reform the delivery of healthcare that can be responsive to patients' needs. The implementation of remote consultations for emergency ophthalmology at Moorfields, both during and beyond the pandemic, also led to a significant reduction in the number of patients attending hospital [13]. Glaucoma monitoring however, is reliant upon diagnostic tests including measurement of visual acuity and IOP, visual field testing and optic nerve imaging which are not yet sufficiently mature to deliver reliably in a home-monitoring setting [14]. The NHS National Outpatient Transformation Programme [15] has therefore highlighted the need to develop community "diagnostic hubs" where such tests can be performed in order to reduce faceto-face consultations in a hospital setting. The successful utilisation of adjunctive telephone consultations to discuss changes in disease status, treatment and future management with patients has been integral to the restructuring of the glaucoma service at Moorfields, which aimed to increase capacity whilst also reducing the need for face-to-face clinic attendances.

Our study also demonstrates that patients who received a telephone consultation prior to their booked appointment are less likely to miss their appointment, compared to those who did not receive a telephone consultation $(13.9 \%$ vs $29.3 \%, p<0.00001)$ leading to increased clinic efficiency on the day. Clinic nonattendance is a complex and multifactorial challenge $[3,16]$ that hospital eye services faced even prior to the pandemic, during which non-attendance rose significantly. All patients at Moorfields are sent text message reminders prior to their appointment, a practice which has been demonstrated to improve clinic attendance rates in a systematic review [17]. Good communication and patient education has also been shown to improve patients' understanding of their condition and enhance adherence with glaucoma treatments [18]. This additional telephone interaction with a clinician, which offered an opportunity to explain the nature and importance of the appointment, likely influenced the observed difference. We also found that a greater proportion of patients who received a telephone consultation telephoned to rebook their appointment for a future date due to concerns about COVID-19 compared to those who did not receive a call $(6.1 \%$ vs $0.7 \%, p<0.0003$ ). This increased interaction as a consequence of the initial call is likely to reduce the future risk of patients being lost to follow up in the long-term.

It is important to note that almost forty-five percent of booked patients did not receive a telephone consultation. This reflects the incomplete contact information held within the hospital PAS, and the fact that patients may have been unwilling to answer a call from an unfamiliar number. This is a potential barrier to the future expansion and widespread implementation of remote monitoring clinics, the success of which is reliant upon an adjunctive telephone consultation. This highlights the importance of NHS providers ensuring that contact details for patients are updated at every interaction.

There are limitations to this study. The relationship between the telephone phone call and non-attendance may be confounded by patient characteristics. There may be bias towards those patients
Table 5. Incidence of clinical observations indicating increased risk and treatment escalations.

\begin{tabular}{lc|}
\hline Details of event & Number (\%) \\
\hline Total events & $35(7.3)$ \\
\hline IOP $\geq 30 \mathrm{mmHg}$ (either eye) & $17(3.5)$ \\
\hline $\mathrm{IOP} \geq 40 \mathrm{mmHg}$ (either eye) & $6(1.3)$ \\
\hline Loss of $\geq 2$ lines of Snellen Visual Acuity (either eye) & $12(2.5)$ \\
\hline Treatment escalations & Number (\%) \\
\hline Total escalations & $50(10.4)$ \\
\hline Change/Increase in glaucoma medications & $29(6.1)$ \\
\hline Booked for selective laser trabeculoplasty & $13(2.7)$ \\
\hline Booked for cataract surgery & $5(1.0)$ \\
\hline Booked for glaucoma surgical intervention & $3(0.6)$
\end{tabular}

who owned mobile telephones or who were more confident in spoken English. In order to truly ascertain the safety of RGACs, it would be necessary to study the long-term outcomes of patients triaged by this pathway and assess the risk of harm and the incidence of preventable sight loss. RGACs are not a replacement for conventional glaucoma assessments which would include perimetry and optic disc imaging. However, there is little doubt that it is safer for "low-risk" patients to be seen in a RGAC to enable more accurate risk stratification, than to not be seen at all.

In summary, RGACs can safely and effectively deliver high throughput data-driven clinical assessments for the tens of thousands of patients stratified as "low-risk" following deferral of glaucoma outpatient appointments due to the COVID-19 pandemic. Focus on simple, rapidly acquired 'red-flag' markers of possible harm and risk factors for harm, enables identification and treatment of those patients most at risk during the recovery period thus reducing preventable vision loss.

\section{Summary}

What was known before

- The provision of timely care to the high volume of glaucoma patients stratified as low risk following pandemic-related appointment deferrals continues to prove challenging for glaucoma specialists.

- It is unknown whether stratification as low risk remains valid over time, raising the potential risk of harm during this period if left unmonitored.

What this study adds

- Rapid Glaucoma Assessment Clinics are an effective approach to deliver high throughput clinical assessments for large numbers of low-risk glaucoma patients with deferred appointments.

- They enable the rapid identification and treatment of patients who would otherwise face significantly delayed review, thus reducing the risk of future preventable vision loss.

\section{REFERENCES}

1. Rahman F, Zekite A, Bunce C, Jayaram H, Flanagan D. Recent trends in vision impairment certifications in England and Wales. Eye. 2020;34:1271-8.

2. The Way Forward: Options to help meet demand for the current and future care of patients with eye disease. London: Royal College of Ophthalmologists; 2017.

3. Davis A, Baldwin A, Hingorani M, Dwyer A, Flanagan D. A review of 145,234 ophthalmic patient episodes lost to follow-up. Eye. 2017;31:422-9. 
4. Foot B, MacEwen C. Surveillance of sight loss due to delay in ophthalmic treatment or review: frequency, cause and outcome. Eye. 2017;31:771-5.

5. Lack of timely monitoring of patients with glaucoma. Healthcare Safety Investigation 2019/001. United Kingdom: Healthcare Safety Investigation Branch; 2020.

6. Jayaram H, Strouthidis NG, Gazzard G. The COVID-19 pandemic will redefine the future delivery of glaucoma care. Eye. 2020;34:1203-5.

7. Gunn PJG, Marks JR, Au L, Waterman H, Spry PGD, Harper RA. Acceptability and use of glaucoma virtual clinics in the UK: a national survey of clinical leads. BMJ Open Ophthalmol. 2018;3:e000127.

8. Kotecha A, Brookes J, Foster PJ. A technician-delivered 'virtual clinic' for triaging low-risk glaucoma referrals. Eye. 2017;31:899-905.

9. Standards for virtual clinics in glaucoma care in the NHS hospital eye service. Ophthalmic Services Guidance. London: Royal College of Ophthalmologists; 2016.

10. Joint RCOphth and UKEGS glaucoma risk stratification tool. London: Royal College of Ophthalmologists; 2020.

11. Clinical guide for the management of remote consultations and remote working in secondary care during the coronavirus pandemic. National Institute for Health and Care Excellence, NHS England \& NHS Improvement; 2020. https://www.nice. org.uk/media/default/about/covid-19/specialty-guides/specialty-guide-virtualworking-and-coronavirus.pdf.

12. Golash V, Athwal S, Khandwala M. Teleophthalmology and COVID-19: the patient perspective. Future Health J. 2021;8:e54-e59.

13. Kilduff $C L$, Thomas AA, Dugdill J, Casswell EJ, Dabrowski $M$, Lovegrove $C$, et al. Creating the Moorfields' virtual eye casualty: video consultations to provide emergency teleophthalmology care during and beyond the COVID-19 pandemic. BMJ Health Care Inform. 2020;27:e100179. https://doi.org/10.1136/bmjhci-2020-100179.

14. Strouthidis NG, Chandrasekharan G, Diamond JP, Murdoch IE. Teleglaucoma: ready to go? Br J Ophthalmol. 2014;98:1605-11.

15. Transforming NHS outpatient care: national outpatient transformation programme. NHS England; 2020.

16. King A, David D, Jones HS, O'Brien C. Factors affecting non-attendance in an ophthalmic outpatient department. J R Soc Med. 1995;88:88-90.

17. Gurol-Urganci I, de Jongh T, Vodopivec-Jamsek V, Atun R, Car J. Mobile phone messaging reminders for attendance at healthcare appointments. Cochrane Database Syst Rev. 2013;12:CD007458.

18. Carpenter DM, Blalock SJ, Sayner R, Muir KW, Robin AL, Hartnett ME, et al. Communication predicts medication self-efficacy in glaucoma patients. Optom Vis Sci. 2016;93:731-7.

\section{ACKNOWLEDGEMENTS}

GG is employed by UCL and supported by grants from the National Institute for Health Research (HTA 09/104/40), Moorfields Eye Charity, British Council to Prevent Blindness, Fight For Sight and the International Glaucoma Association. $\mathrm{HJ}$ is supported by the Moorfields Eye Charity. GG and HJ are grateful for the support of the National Institute for Health Research Biomedical Research Centre for Ophthalmology at Moorfields Eye Hospital and the UCL Institute of Ophthalmology. The views expressed in this paper are those of the authors and not necessarily those of any funding body or the UK Department of Health.

\section{AUTHOR CONTRIBUTIONS}

$A B \& H J$ carried out the data collection and analysis. $H J, A B \& G G$ contributed to the first draft and all authors approved the final version of the manuscript. All authors agree to be accountable for all aspects of the work in ensuring that questions related to the accuracy or integrity of any part of the work are appropriately investigated and resolved.

\section{COMPETING INTERESTS}

The authors declare no competing interests.

\section{ADDITIONAL INFORMATION}

Correspondence and requests for materials should be addressed to H.J.

Reprints and permission information is available at http://www.nature.com/ reprints

Publisher's note Springer Nature remains neutral with regard to jurisdictional claims in published maps and institutional affiliations. 